\title{
Effects of FGF-2 release from a hydrogel polymer on bone mass and microarchitecture
}

\author{
Guillaume Mabilleau a , Eric Aguado ${ }^{\mathrm{a}, \mathrm{b}}$, Izabella C. Stancu ${ }^{\mathrm{a}, \mathrm{c}}$, \\ Corneliu Cincu $^{\text {a,c }}$, Michel F. Baslé ${ }^{a}$, Daniel Chappard ${ }^{\mathrm{a}, *}$ \\ ${ }^{a}$ INSERM, U922 - LHEA, Faculté de Médecine, 49045 Angers Cedex, France \\ ${ }^{\mathrm{b}}$ BBTOCEX, Ecole Nationale Vétérinaire, 1 route de Gachet, 44307 Nantes, France \\ ${ }^{\mathrm{c}}$ Department of Macromolecular Chemistry, Industrial Chemistry Faculty, University Politehnica, 71101 Bucharest, Romania \\ Received 9 October 2007; accepted 15 December 2007 \\ Available online 22 January 2008
}

\begin{abstract}
Bone substitutes are widely used for filling and restoring bone defects. Among them, methacrylic polymers are employed in load-bearing bones to seal hip prostheses. Incorporation of growth factors into a polymer device could be a way to enhance bone growth. In the present study, we evaluated the capacity of poly(2-hydroxyethyl methacrylate) - pHEMA - copolymerized with 2-vinyl pyrrolidone - VP - to release proteins. Fibroblast growth factor-2 (FGF-2) was incorporated into cylinders of p(HEMA-co-VP). FGF-2 release was studied by ELISA in vitro and cylinders were implanted in the femoral condyle of white New Zealand rabbits. After 2 months post-surgery, FGF-2 was able to enhance bone formation by increasing bone volume; this effect was evidenced by an increase in trabecular number and bone gain was mainly in the form of woven bone. At 3 months post-surgery, no difference could be evidenced between animals receiving vehicle or FGF-2. Animals receiving vehicle exhibited bone mass higher than at 2 months and woven bone was replaced by mature bone with a lamellar matrix. The hydrogel polymer allowed the release of FGF-2, which in return enhanced bone regeneration soon after surgery but the effect vanished rapidly.
\end{abstract}

(C) 2007 Elsevier Ltd. All rights reserved.

Keywords: Controlled release; FGF-2; Histomorphometry; Growth factor; pHEMA

\section{Introduction}

Bone substitutes are materials used for filling and restoring bone defects. The use of growth factors incorporated into bone substitutes may be one possibility to enhance bone integration and remodeling [1,2]. Fibroblast growth factors (FGFs) are a family of polypeptides that control proliferation and differentiation of various cell types [3,4]. FGFs are also strong inducers of angiogenesis and important effects on the control of osteoblast differentiation genes have been recently recognized [5]. FGF-2 is a member of this family which acts actively in the skeletal development [6]. In bone fetus, FGF-2 transcripts are found in mesenchymal cells and osteoblasts

\footnotetext{
* Corresponding author. Tel.: +33 241735865 .

E-mail address: daniel.chappard@univ-angers.fr (D. Chappard).
}

$[7,8]$. In the postnatal life, FGF-2 is produced by mature osteoblasts and stored in the bone matrix [9]. It acts as a potent mitogen of bone cells in culture but the effect of FGF-2 on the healing of bone defects remains controversial [10]. The two main ways of administration of FGF-2 are systemic and subcutaneously injections. However, the recent finding that systemic injections of FGF-2 could provoke extra skeletal effects led to find others ways of administration [11]. Local delivery by a synthetic polymer could represent a good way of administration in patients needing bone reconstruction. The most important parameter to take into account is the release kinetic of FGF-2 from the carrier: Tabata et al. showed that the controlled release of FGF-2 from gelatin hydrogels promotes a better skull bone regeneration than the single injection of the same dose [12]. A hydrogel with a low degradation rate would sustain the formation activity of osteoblasts in bone defects. For the reconstruction of load-bearing bones, the use 
of a polymer with biomechanical properties similar to bone is an additional prerequisite [13]. Poly(2-hydroxyethyl methacrylate) (pHEMA) appears to be a good candidate for the controlled release of growth factors in bone. It is a poorly swellable hydrogel which can be polymerized with other monomers to improve its properties [14]. pHEMA is erodible by macrophages but the degradation rate is low [15]. $N$-vinyl2-pyrrolidone (VP) is a hydrophilic monomer able to polymerize with HEMA to obtain a drug delivery device [16]. The copolymer p(HEMA-co-VP), with a ratio respectively 40:60, was used to modulate the release of cyclosporine in soft tissue [17]. Moreover, the addition of HEMA and VP in bone cement improves the release of antibiotics [18].

The aim of this study was to use p(HEMA-co-VP) as a copolymer for the reconstruction of a bone defect in a loadbearing bone. We have used a ratio HEMA:VP of 80:20 to prepare a moderately swollen hydrogel having a slow degradation rate. Cylinders of the copolymer loaded with FGF-2 were implanted in a hole drilled in the right femoral condyle of white New Zealand rabbits. The effects of FGF-2 on bone mass and microarchitecture were investigated by microcomputed tomography (microCT) and the quality of the new bone matrix was assessed by histomorphometric analysis after toluidine blue staining.

\section{Materials and methods}

All chemical reagents were obtained from Sigma-Aldrich Chemical (Illkirsh, France). Commercial 2-hydroxyethyl methacrylate (HEMA) contains impurities due to the fabrication process. The polymerization inhibitor 4-methoxyphenol (added by the manufacturer before shipping, at a concentration of $350 \mathrm{ppm}$ ) also needs to be removed. HEMA and vinyl pyrrolidone (VP) were purified and distilled under reduced pressure. Benzoyl peroxide (BPO) was recrystallized in methanol two times before use to remove impurities.

\subsection{Immobilization process of growth factor}

Human FGF-2 obtained from Chemicon International Inc. (Temecula, California) was incorporated into the polymer according to our previously described method [19]. Briefly, $550 \mu \mathrm{g}$ of FGF-2 was dissolved in $100 \mu \mathrm{l}$ of PBS and the aqueous colloidal solution was added to $2.5 \mathrm{~g}$ of a mixture composed of HEMA (80\%) and VP (20\%) containing $0.125 \%(\mathrm{w} / \mathrm{v})$ of $\mathrm{BPO}$, a polymerization accelerator. The growth factor was homogenized by vortexing for $5 \mathrm{~min}$. In parallel, tubes containing the same amount of PBS were used to prepare the control polymer. The polymerization initiator $\mathrm{N}, \mathrm{N}$ dimethyl paratoluidine was added at a concentration of $0.03 \%$ and the mixture was vortexed again for $5 \mathrm{~min}$. The accelerated and initiated mixture was poured into silicon tubes and polymerized at $4{ }^{\circ} \mathrm{C}$ for $24 \mathrm{~h}$. Tubes of $\mathrm{p}$ (HEMA-co-VP) were collected and sectioned into cylinders (4-mm in diameter and 6-mm in length) with a razor blade. Each cylinder contained approximately $45 \mu \mathrm{g}$ of FGF-2. Cylinders were sterilized under ultraviolet radiation $(360 \mathrm{~nm}$ for $3 \mathrm{~h}$ ) before use. This method of sterilization is enough to avoid microbial contamination, as we have reported earlier [19].

\subsection{Swelling of the copolymer in vitro}

Swelling behavior was evaluated by the blot-and-weight technique. Cylinders were weighted to determine the dry weight $\left(W_{\mathrm{d}}\right)$ and then allowed to swell in saline, at constant temperature $\left(22^{\circ} \mathrm{C}\right)$ for 2 months. Experiments were done in triplicate. After immersion at different incubation intervals, cylinders were blotted to remove saline excess and weighted to evaluate the wet weight $\left(W_{\mathrm{w}}\right)$. After measuring, cylinders were deposed in saline to carry on the swelling process. For each type of polymer, the swelling degree was evaluated using the equation:

Swelling degree $=\frac{W_{\mathrm{w}}-W_{\mathrm{d}}}{W_{\mathrm{d}}} \times 100$

\subsection{In vitro release of $F G F-2$}

Six cylinders, containing $45 \mu \mathrm{g}$ of FGF-2, were allowed to swell in $2 \mathrm{ml}$ of phosphate buffer saline (PBS) during 1 week at $37^{\circ} \mathrm{C}$ in a humidified atmosphere. Every day, all the PBS were removed and stored at $-20^{\circ} \mathrm{C}$ before assay. The amount of FGF-2 in each sample was determined in triplicate by ELISA, using the Quantikine kit from R\&D systems (Lille, France). Absorbance was measured at $450 \mathrm{~nm}$ on a microplate reader.

\subsection{Animals and surgical procedure}

Twenty-four female New Zealand rabbits (approximate weight $3.5 \mathrm{~kg}$ ) were randomly divided into two groups:

- 12 animals received pHEMA cylinders containing the vehicle and constituted the control group. Six rabbits were sacrificed 2 months after surgery and the remaining six at 3 months post-surgery.

- 12 rabbits received pHEMA cylinders containing vehicle + FGF-2 and constituted the FGF group. Six rabbits were sacrificed 2 months after surgery and the remaining six at 3 months.

Bilateral femoral implantations were performed under aseptic conditions and general anesthesia. After skin incisions and lateral arthrotomy access via the knee joint, a cylindrical defect ( $4 \mathrm{~mm}$ diameter and $6 \mathrm{~mm}$ long) was created at the distal femoral end and then filled with pHEMA cylinders. The cylinder was placed after the cavity had been flushed with sterile saline to remove debris. Incision was closed by different layers with resorbable sutures. The animals were killed 2 and 3 months after implantation by an intracardiac overdose of sodium pentobarbital (Dolethal, Vetoquinol, France). The lower femoral extremities were immediately dissected from the animal and fixed in $10 \%$ formalin.

The care and use of these laboratory animals were in compliance with French law on animal experimentation. Implantation control was done to be ensuring that biomaterials have been implanted in the right position. X-ray examination was done 3 days after implantation. Radiographs of the right hind limb were performed using a Faxitron X-ray system (MX20, Edimex, Angers, France) with a $5 \times 5 \mathrm{~cm}$ CCD camera. The accelerating voltage was fixed at $35 \mathrm{kV}$ with a $15 \mathrm{~s}$ exposure time. X-ray images were taken at 1,2 and 3 months after implantation.

\subsection{X-ray microcomputed tomography}

MicroCT was done with a Skyscan 1072 microtomograph (Skyscan, Kontich, Belgium). Analysis of bone samples was done with the cone beam procedure. 3D modeling and analysis reconstruction of the bone were obtained with the ANT software (release 2.2.6; Skyscan). The program allows reconstruction of the object from the stack of 2D sections, after interactive segmentation. The reconstructed 3D models were obtained by a surface-rendering algorithm. The bone volume and architecture parameters on the 3D models were appreciated on a zone of $14 \mathrm{~mm}$ in height (including $5 \mathrm{~mm}$ above the implant, the implant itself and $5 \mathrm{~mm}$ below the implant as the goal of the study was to assess the bone regeneration at the margin of the implant) and were determined with a dedicated software (CtAN) release 1.3.2.2. Trabecular bone volume (BV/TV, in \%), trabecular number (Tb.N, in $/ \mathrm{mm}$ ), trabecular separation $(\mathrm{Tb} . \mathrm{Sp}$, in $\mu \mathrm{m})$, trabecular thickness ( $\mathrm{Tb} . \mathrm{Th}$, in $\mu \mathrm{m})$, trabecular bone pattern factor $\left(\mathrm{Tb} . \mathrm{P}_{\mathrm{f}}\right)$ and the structure model index (SMI) were calculated from 3D models of the femoral condyle. Tb. $\mathrm{P}_{\mathrm{f}}$ is mainly based on the use of mathematical morphology in image analyzer systems. The rationale of the method is supported by the observation that, in a well-connected structure, the concave surfaces are abundant whereas convex surfaces are more numerous if the structure is disconnected. This method provides low values 
of Tb. $\mathrm{P}_{\mathrm{f}}$ in a well-connected network and high values when marked disconnection of trabeculae is present [20]. SMI characterizes a 3D bone structure composed of a certain amount of plates and rods. SMI has a value between 0 and 3. In an ideal plate structural model, the SMI value is 0 , and in an ideal cylindrical rod structure, SMI is 3 , independent of the physical dimensions of the structure [21].

\subsection{Histological procedure}

Specimens were prepared undecalcified as described elsewhere [22]. Briefly, bone samples were immersed in $10 \%$ formalin for 3 days at $4{ }^{\circ} \mathrm{C}$. They were dehydrated in absolute acetone for $72 \mathrm{~h}$ at $4{ }^{\circ} \mathrm{C}$ (three changes), defatted in xylene, and embedded in purified methyl methacrylate. Longitudinal sections ( $300 \mu \mathrm{m}$ in thickness) were made parallel to the long axis of the bone on a contact-point saw equipped with a diamond band (Exakt 310, Norderstedt, Germany). Slices were affixed onto plastic translucent slides with cyanoacrylic glue. They were ground to a thickness of $\sim 30 \mu \mathrm{m}$ on a bench-top grinder with silicon carbide papers ranging from 220 to 4000 (Struers, Champigny sur Marne, France). Sections were surface-stained with toluidine blue (1\% in sodium tetraborax) for histological study and quantitative analysis. This staining allows a clear identification of the woven bone: toluidine blue is highly fixed by glycosaminoglycans present in large amounts in woven bone and in low amounts in lamellar bone [23]. Digitized microphotographs were taken around the implant and the woven bone volume (WB.Ar/T.Ar, expressed in \%) was measured using ImageJ release 1.34s (NIH, Bethesda, MD).

\subsection{Statistical analysis}

Statistical analysis was performed using Systat ${ }^{\circledR}$ statistical software release 11.0 (SPSS Inc., Chicago, Illinois). A non-parametric one-way analysis of variance (Kruskal-Wallis ANOVA) was used for comparing the differences between groups. Differences were considered as significant when $p<0.05$. Results are expressed as mean \pm standard error of the mean (SEM).

\section{Results}

\subsection{Protein encapsulation}

Bulk polymerization was achieved in $24 \mathrm{~h}$ under experimental conditions providing hard pHEMA cylinders on the whole tube height. Cylinders had a light yellow tint and were free of bubbles.

\subsection{Release of FGF-2 from $p(H E M A-c o-V P)$ cylinders in vitro}

The release of FGF-2 from p(HEMA-VP) hydrogel was followed by ELISA. Our data suggested that human FGF-2 is released from the copolymer during the swelling process and was neither damaged by the polymerization nor the sterilization processes (Fig. 1a). Human FGF-2 appeared to be released in two times: during the first 4 days, human FGF-2 was released slowly with roughly $1 \%$ of the total amount loaded in the copolymer per day. Then, after 5 days, the release of human FGF-2 increased to reach $3.5 \%$ per day. Interestingly, the swelling of the copolymer indicated that during the first 5 days, the polymer reached only $50 \%$ of its total swelling (Fig. 1b).

\subsection{Control of implantation by X-ray}

On X-ray images, the drilled hole appeared as a dark spot throughout the femoral condyle because the polymer is
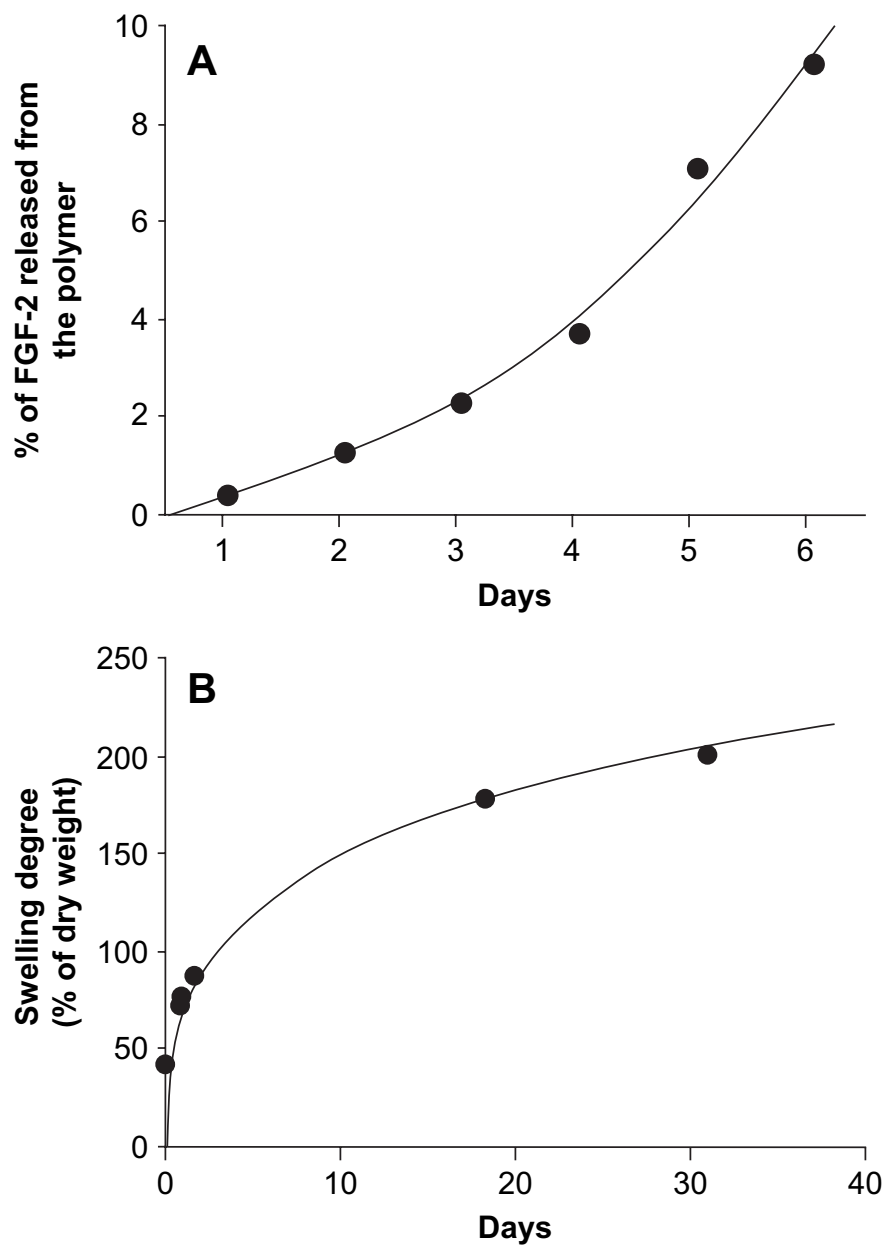

Fig. 1. (A) Amount of FGF-2 released from polymer cylinders over time. (B) Swelling behavior of the $\mathrm{p}$ (HEMA-co-VP) copolymer.

radiolucent. After 2 months post-surgery, a white calcified condensation appeared around the hole filled with the polymer as a radio-opaque ring. This thin collar of newly formed bone was evidenced on radiographic images irrespective of the X-ray beam angulation. This aspect persisted after 3 months in animals from control and FGF groups (Fig. 2).

\subsection{Bone mass and microarchitecture \\ 2 months post-surgery}

MicroCT evidenced the formation of a peripheral bone layer all around the polymer surface which corresponds to the radio-opaque ring observed on radiographs. This bone layer corresponded to the peripheral densification observed on radiographs. A direct bone bonding was observed both in control and FGF rabbits and the bony ring appeared composed of a delicate lace of small bone trabeculae. The ring was thicker in FGF animals (Fig. 3). Animals from the FGF group showed a significant increase in bone mass compared to control group as evidenced by higher values for BV/TV $(+75.8 \%)$ and Tb.N (+60\%) (Table 1). Modifications of the microarchitecture were evidenced by a significant decrease in $\mathrm{Tb} . \mathrm{P}_{\mathrm{f}}$ $(-57.5 \%)$ and SMI $(-40.7 \%)$ indicating a better connected 

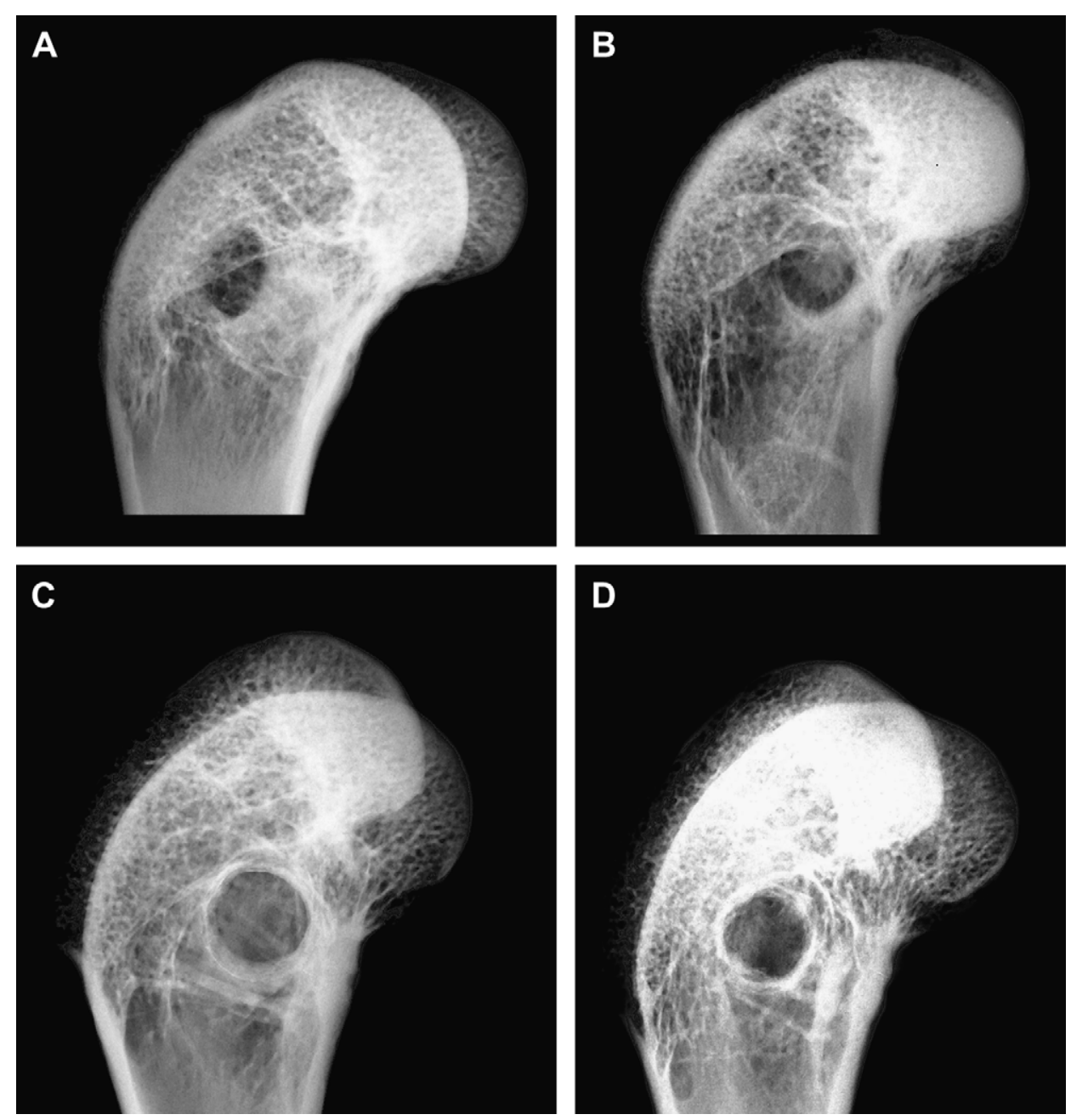

Fig. 2. X-ray images of control and FGF groups. (A) Control group at 2 months post-surgery, (B) FGF group at 2 months post-surgery, (C) Control group at 3 months post-surgery, and (D) FGF group at 3 months post-surgery. Note the presence of a white calcified area around the hole filled with the polymer.

network with more trabeculae in the form of plate than pillar; animals from control groups exhibited a prevalence of pillars. No significant difference was noted for Tb.Th and Tb.Sp. Histological sections confirmed the presence of a bony ring formed at the surface of the polymer in both groups (Fig. 4a and $\mathrm{b}$, respectively) and the bony ring was thicker $(+93.7 \%)$ in the FGF group (Fig. 5). The volume of woven bone was 7.2-fold higher in the FGF group. Packets of newly formed bone were observed in the vicinity of the polymer cylinder but also at distance from the implantation site in the FGF group. Polymer particles were sometimes found enclosed in trabeculae in both groups.

\subsection{Bone mass and microarchitecture, \\ 3 months post-surgery}

MicroCT also identified the bony ring around the cylinders but no difference could be observed between groups. Animals from the control group exhibited a higher bone mass at 3 than at 2 months. BV/TV increased from $9.88 \pm 1.44$ to $16.34 \pm 5.8$ and Tb.N from $0.49 \pm 0.05$ to $0.80 \pm 0.19$ (Table 1). No significant change was noted for $\mathrm{Tb}_{\mathrm{f}}$, SMI and Tb.Sp between 2 and 3 months. Histological sections still revealed the persistence of the bony ring around the implant. Thickness of the bony ring was increased $(+88.27 \%)$ and was similar to the thickness of the ring in the FGF group at 2 months (Fig. $4 \mathrm{~b}$ and Fig. 5). A significant decrease in the amount of woven bone around the biomaterial $(-49.8 \%)$ was noted. Animals from the FGF group did not exhibit significant differences in histomorphometric parameters when compared to control animals (Table 1). The only noticeable difference, when compared with 2 months data, concerned woven bone volume, with a significant decrease $(-93.6 \%)$. Here again, particles of polymer were found trapped into bone trabeculae in both control and FGF animals.

\section{Discussion}

Growth factors were actively tried in the last decade to promote bone regeneration [24-26]. Our results indicated that the protein was capable to be released from the polymer during the swelling process. We have shown in several previous 

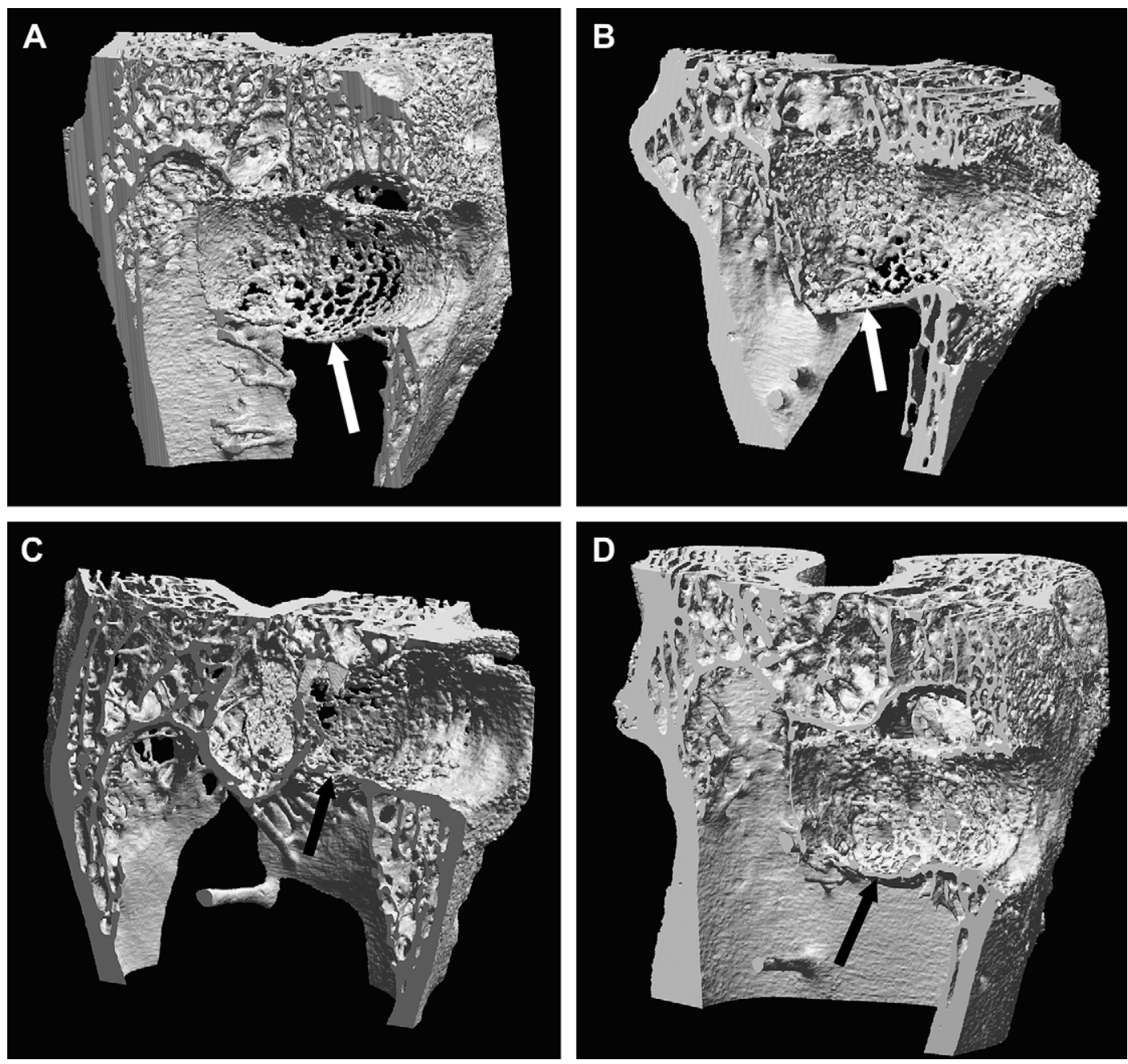

Fig. 3. 3D models obtained with microCT, the bone layer apposed onto the cylinder is identified with an arrow. (A) Control group at 2 months, (B) FGF group at 2 months, (C) control group at 3 months, and (D) FGF group at 3 months. The bony layer apposed is thicker around the FGF cylinders; note the thin lace of trabeculae that are formed at the surface of the polymer.

studies that our method used to entrap proteins during polymerization is non-destructive for the molecule and do not induce any loss of activity $[19,27,28]$. Moreover, the detection of FGF-2 by ELISA exhibited this statement. Administration of FGF-2 was extensively studied in the literature after systemic or subcutaneous injections. Bone anabolic effects are regularly reported: increase in osteoblast surfaces, osteoid surfaces, osteoid volume and a decrease in osteoclast surfaces [29-31]. However, the recent finding that systemic injection of FGF-2 could provoke anemia with an increase in the granulocyte production in the liver and a mild hypertrophy of renal glomeruli, led to find other ways of administration [11]. Local administration of growth factors into bone appeared an interesting solution in patients needing bone filling, to avoid extra skeletal effects. In the present study, the anabolic effect of FGF-2 release on bone mass was evidenced after 2 months post-surgery. A significant increase in trabecular bone mass and microarchitecture modifications was noted, mainly due to a greater number of trabeculae (evidenced by a higher Tb.N). Trabecular thickness showed a tendency to increase in the FGF group but the difference with control was not significant. At 3 months post-surgery, no difference between control group and FGF group could be noted. Control animals had an increase in bone mass which reached the level of FGF group at 2 months. On the other hand, FGF-2 group at 3 months preserved the bone mass and architecture parameters reached at 2 months. The only parameter changed is the woven bone area, which is replaced by lamellar bone, in control and FGF groups. However, woven bone is poorly mineralized and has low mechanical properties; it is a transient state that must be replaced by lamellar bone to obtain satisfying stiffness and toughness [32]. The present study demonstrated that a local delivery of FGF-2 facilitated the bone regeneration from the edge of the defect. Niedhart et al. have used a tricalcium phosphate cement to deliver FGF-2 during 1 and 3 weeks [2]. They were not able to identify a difference between the cement alone and the cement loaded with FGF-2. They concluded that FGF-2 was released too slowly or too fast from the cement. An important aspect is the kinetic of release of FGF-2 from the carrier. Tabata 
Table 1

Histomorphometrical parameters of control and FGF groups

\begin{tabular}{lccccr}
\hline Parameters & \multicolumn{2}{l}{ Control group } & \multicolumn{3}{l}{ FGF group } \\
\cline { 2 - 3 } \cline { 5 - 6 } \cline { 5 - 6 } & 2 months & 3 months & & 2 months & 3 months \\
\hline BV/TV (\%) & $9.88 \pm 1.44$ & $16.34 \pm 5.80^{\mathrm{b}}$ & & $17.37 \pm 3.7^{\mathrm{a}}$ & $16.34 \pm 3.97$ \\
Tb.N $(/ \mathrm{mm})$ & $0.50 \pm 0.06$ & $0.80 \pm 0.2^{\mathrm{b}}$ & & $0.80 \pm 0.12^{\mathrm{a}}$ & $0.84 \pm 0.17$ \\
Tb.Th $(\mu \mathrm{m})$ & $0.20 \pm 0.01$ & $0.20 \pm 0.03$ & & $0.21 \pm 0.02$ & $0.19 \pm 0.01$ \\
Tb.Sp $(\mu \mathrm{m})$ & $1.68 \pm 0.26$ & $1.43 \pm 0.15$ & & $1.74 \pm 0.35$ & $1.43 \pm 0.19$ \\
Tb.P & $7.75 \pm 1.10$ & $4.70 \pm 3.07$ & & $3.29 \pm 1.61^{\mathrm{a}}$ & $4.61 \pm 2.13$ \\
SMI & $2.26 \pm 0.10$ & $1.56 \pm 0.57$ & & $1.34 \pm 0.30^{\mathrm{a}}$ & $1.59 \pm 0.38$ \\
WB.Ar/T.Ar & $0.24 \pm 0.11$ & $0.12 \pm 0.02^{\mathrm{b}}$ & $1.73 \pm 0.50^{\mathrm{a}}$ & $0.11 \pm 0.04^{\mathrm{b}}$ \\
$\quad(\%)$ & & & & & \\
B ring.Th & $66.99 \pm 4.50$ & $126.13 \pm 9.45^{\mathrm{b}}$ & & $129.77 \pm 6.15^{\mathrm{a}}$ & $110.52 \pm 6.94$
\end{tabular}

${ }^{\text {a }} p<0.05$ vs. control group at the same month post-surgery.

b $p<0.05$ vs. 2 months.

et al. showed that free FGF-2 given into an osseous defect without carrier induced significantly less bone ingrowth compared with carrier-associated FGF-2 [12]. They showed that gelatin is a good carrier for FGF-2 in skull bone defect. Radomsky et al. have used hyaluronan to encapsulate FGF-2 [33]. They showed that the combination of hyaluronan and FGF-2 accelerated fracture healing in an experimental primate fracture model. But, for the reconstruction of load-bearing bones, the use of a polymer with biomechanical properties similar to bone is an additional prerequisite [13]. In the present study $\mathrm{p}$ (HEMA-co-VP) appeared a good candidate for the controlled release of growth factors in load-bearing bones. The rate of release appeared compatible with the kinetic of action of FGF-2. Presence of polymer particles inside the bone trabeculae indicated that polymer could be erodable and bone bounded. The persistence of the cylinder after 3 months confirmed that pHEMA is slowly degradable and this finding is in agreement with previous data [15].

There exists several ways of delivery from a biomaterial: hydrophobic biomaterials like poly(lactic acid) release their content by degradation [34]. Hydrogels are a category of biomaterials which can swell; the relaxation of the macromolecular chains allows the release of entrapped factors. pHEMA presents hardness (expressed in Shore D unit, usually employed to assess polymer hardness [35]) lower than bovine cortical bone $(85.58 \pm 1.53$ Sh.D vs. $63.278 \pm 1.617$ Sh.D). However, to achieve desired properties (biomechanical, swelling...), pHEMA can be copolymerized with others monomers, plasticizers and cross-linking agents. In a previous study, we have reported that cross-linked pHEMA (with tetraethylene glycol diacrylate or polyethylene glycol diacrylate) has a Young's modulus and hardness close to bone [35]. Moreover only these cross-linkers with a long backbone separating the two acrylate groups allow the swelling of the hydrogel close to the linear form of pHEMA. In the present study, 2-vinyl pyrrolidone appeared suitable to enhance release properties of pHEMA.
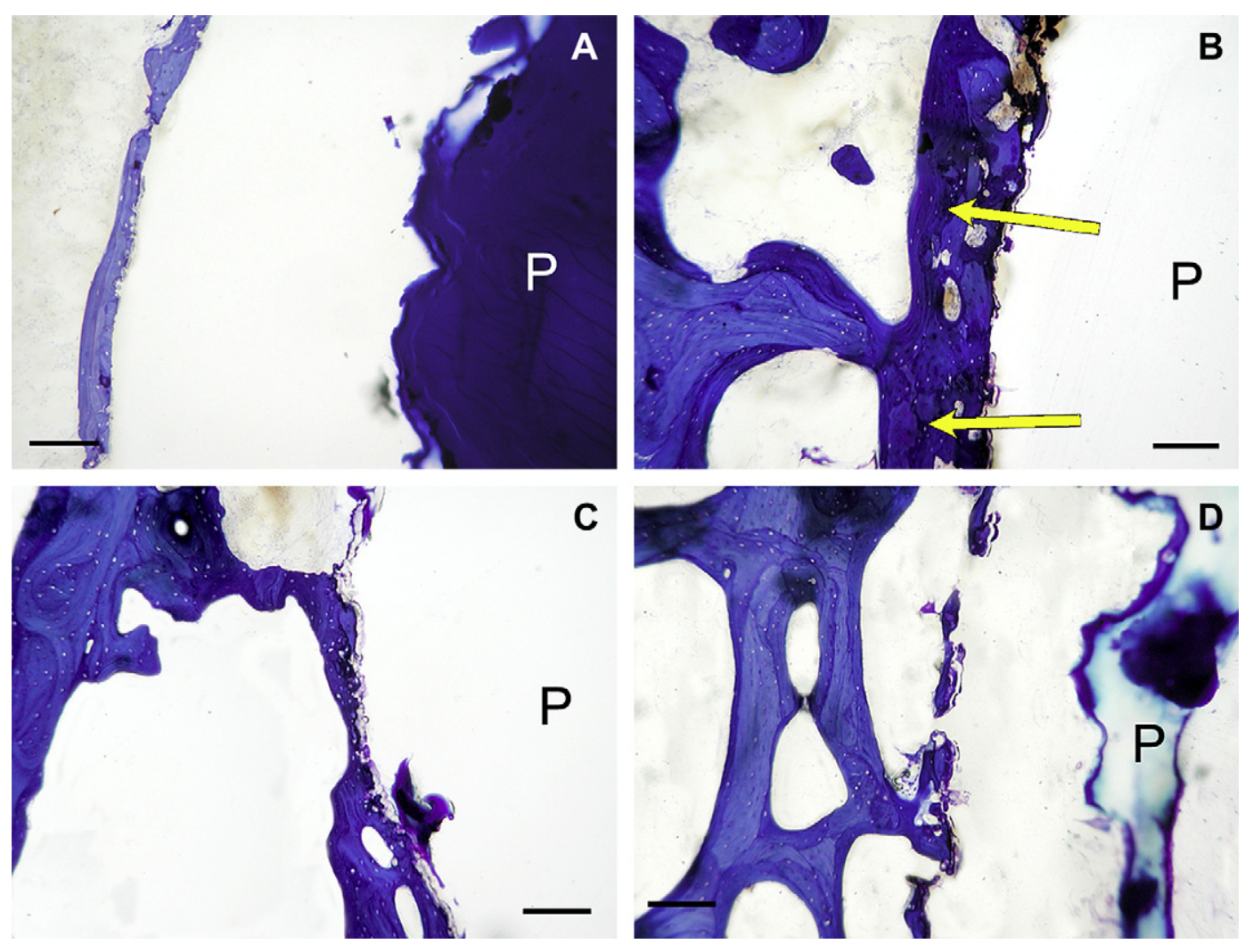

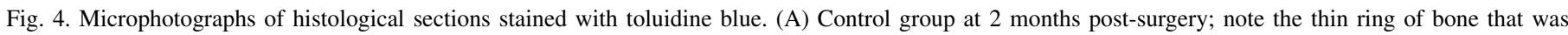

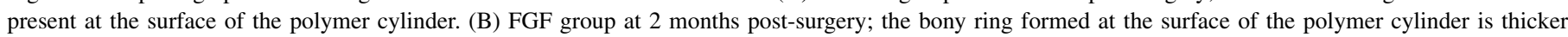

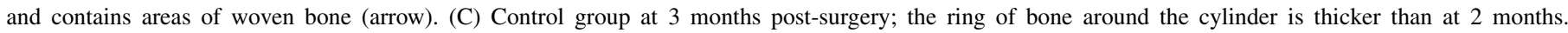

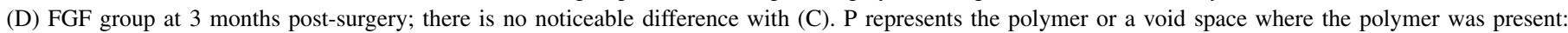
the hydrogel shrinks considerably during the histological process in the solvents used during the histotechnological steps. Bar represents $250 \mu \mathrm{m}$. 


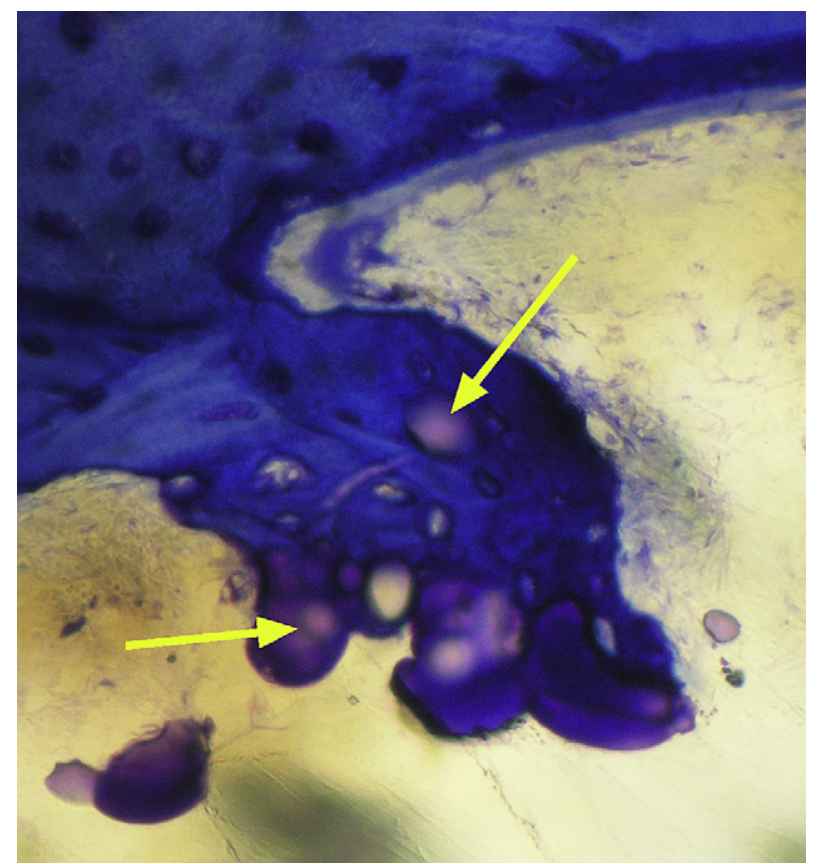

Fig. 5. Microphotographs of histological sections stained with toluidine blue. Note that some polymer particles are directly covered by bone (arrows).

\section{Conclusion}

The controlled release of FGF-2 from a copolymer $\mathrm{p}(\mathrm{HEMA}-\mathrm{co}-\mathrm{VP})$ stimulated the bone regeneration at the edge of the defect. However, the effects of FGF-2 appeared to vanish rapidly after 2 months post-surgery. The copolymer appeared suitable for the controlled release of growth factor into load-bearing bone needing a fast regeneration.

\section{Acknowledgments}

Authors are greatly indebted to G. Brossard and N. Gaborit for their help with X-ray microCT. G. Mabilleau received a fellowship from the French Ministry of Research and Technology. This work was made possible by grants from Contrat de Plan Etat - Région "Pays de la Loire" and INSERM.

\section{References}

[1] Mundy GR. Pathogenesis of osteoporosis and challenges for drug delivery. Adv Drug Deliv Rev 2000;42:165-73.

[2] Niedhart C, Maus U, Miltner O, Graber HG, Niethard FU, Siebert CH The effect of basic fibroblast growth factor on bone regeneration when released from a novel in situ setting tricalcium phosphate cement. J Biomed Mater Res A 2004;69:680-5.

[3] Basilico C, Moscatelli D. The FGF family of growth factors and oncogenes. Adv Cancer Res 1992;59:115-65.

[4] Jaye M, Schlessinger J, Dionne CA. Fibroblast growth factor receptor tyrosine kinases: molecular analysis and signal transduction. Biochim Biophys Acta 1992;1135:185-99.

[5] Presta M, Dell'Era P, Mitola S, Moroni E, Ronca R, Rustani M. Fibroblast growth factor/fibroblast growth factor receptor system in angiogenesis. Cytokine Growth Factor Rev 2005;16:159-78.

[6] Naski MC, Ornitz DM. FGF signaling in skeletal development. Front Biosci 1998;3:d781-94
[7] Gonzalez AM, Hill DJ, Logan A, Maher PA, Baird A. Distribution of fibroblast growth factor (FGF)-2 and FGF receptor-1 messenger RNA expression and protein presence in the mid-trimester human fetus. Pediatr Res 1996;39:375-85.

[8] Kim HJ, Rice DP, Kettunen PJ, Thesleff I. FGF-, BMP- and Shh-mediated signalling pathways in the regulation of cranial suture morphogenesis and calvarial bone development. Development 1998; 125:1241-51.

[9] Globus RK, Plouet J, Gospodarowicz D. Cultured bovine bone cells synthesize basic fibroblast growth factor and store it in their extracellular matrix. Endocrinology 1989;124:1539-47.

[10] Globus RK, Patterson-Buckendahl P, Gospodarowicz D. Regulation of bovine bone cell proliferation by fibroblast growth factor and transforming growth factor beta. Endocrinology 1988;123:98-105.

[11] Wamsley HL, Iwaniec UT, Wronski TJ. Selected extraskeletal effects of systemic treatment with basic fibroblast growth factor in ovariectomized rats. Toxicol Pathol 2005;33:577-83.

[12] Tabata Y, Yamada K, Miyamoto S, Nagata I, Kikuchi H, Aoyama I, et al. Bone regeneration by basic fibroblast growth factor complexed with biodegradable hydrogels. Biomaterials 1998;19:807-15.

[13] Moore WR, Graves SE, Bain GI. Synthetic bone graft substitutes. Aust N Z J Surg 2001;71:354-61.

[14] Ratner BD, Hoffman AS, Schoen FJ, Lemons JE. Biomaterials science: an introduction to materials in medicine. 2nd. London: Elsevier Academic Press; 2004.

[15] Mabilleau G, Moreau MF, Filmon R, Basle MF, Chappard D. Biodegradability of poly (2-hydroxyethyl methacrylate) in the presence of the J774.2 macrophage cell line. Biomaterials 2004;25:5155-62.

[16] Blanco MD, Trigo RM, Garcia O, Teijon JM. Controlled release of cytarabine from poly(2-hydroxyethyl methacrylate-co- $N$-vinyl-2-pyrrolidone) hydrogels. J Biomater Sci Polym Ed 1997;8:709-19.

[17] Gallardo A, Fernandez F, Cifuentes A, Diez-Masa JC, Bermejo P, Rebuelta M, et al. Modulated release of cyclosporine from soluble vinyl pyrrolidone-hydroxyethyl methacrylate copolymer hydrogels. A correlation of 'in vitro' and 'in vivo' experiments. J Control Rel 2001;72:1-11.

[18] Frutos P, Diez-Pena E, Frutos G, Barrales-Rienda JM. Release of gentamicin sulphate from a modified commercial bone cement. Effect of (2-hydroxyethyl methacrylate) comonomer and poly( $N$-vinyl-2pyrrolidone) additive on release mechanism and kinetics. Biomaterials 2002;23:3787-97.

[19] Filmon R, Basle MF, Atmani H, Chappard D. Adherence of osteoblastlike cells on calcospherites developed on a biomaterial combining poly(2-hydroxyethyl) methacrylate and alkaline phosphatase. Bone 2002;30:152-8

[20] Hahn M, Vogel M, Pompesius-Kempa M, Delling G. Trabecular bone pattern factor - a new parameter for simple quantification of bone microarchitecture. Bone 1992;13:327-30.

[21] Jinnai H, Watashiba H, Kajihara T, Nishikawa Y, Takahashi M, Ito M. Surface curvatures of trabecular bone microarchitecture. Bone 2002;30:191-4

[22] Grizon F, Aguado E, Hure G, Basle MF, Chappard D. Enhanced bone integration of implants with increased surface roughness: a long term study in the sheep. J Dent 2002;30:195-203.

[23] Haines RW, Mohuiddin A. Metaplastic bone. J Anat 1968;103:527-38.

[24] Kawaguchi H, Kurokawa T, Hanada K, Hiyama Y, Tamura M, Ogata E, et al. Stimulation of fracture repair by recombinant human basic fibroblast growth factor in normal and streptozotocin-diabetic rats. Endocrinology 1994;135:774-81.

[25] Luppen CA, Blake CA, Ammirati KM, Stevens ML, Seeherman HJ, Wozney JM, et al. Recombinant human bone morphogenetic protein-2 enhances osteotomy healing in glucocorticoid-treated rabbits. J Bone Miner Res 2002;17:301-10.

[26] Weiss S, Baumgart R, Jochum M, Strasburger CJ, Bidlingmaier M. Systemic regulation of distraction osteogenesis: a cascade of biochemical factors. J Bone Miner Res 2002;17:1280-9.

[27] Filmon R, Basle MF, Barbier A, Chappard D. Poly(2-hydroxy ethyl methacrylate)-alkaline phosphatase: a composite biomaterial allowing 
in vitro studies of bisphosphonates on the mineralization process. J Biomater Sci Polym Ed 2000;11:849-68.

[28] Filmon R, Basle MF, Barbier A, Chappard D. In vitro study of the effect of bisphosphonates on mineralization induced by a composite material: poly 2(hydroxyethyl) methacrylate coupled with alkaline phosphatase. Morphologie 2000;84:23-33.

[29] Iwaniec UT, Magee KA, Mitova-Caneva NG, Wronski TJ. Bone anabolic effects of subcutaneous treatment with basic fibroblast growth factor alone and in combination with estrogen in osteopenic ovariectomized rats. Bone 2003;33:380-6.

[30] Liang H, Pun S, Wronski TJ. Bone anabolic effects of basic fibroblast growth factor in ovariectomized rats. Endocrinology 1999;140:5780-8.

[31] Nakamura K, Kurokawa T, Aoyama I, Hanada K, Tamura M, Kawaguchi H. Stimulation of bone formation by intraosseous injection of basic fibroblast growth factor in ovariectomised rats. Int Orthop 1998;22:49-54.
[32] Chappard D, Aguado E, Hure G, Grizon F, Basle MF. The early remodeling phases around titanium implants: a histomorphometric assessment of bone quality in a 3- and 6-month study in sheep. Int J Oral Maxillofac Implants 1999;14:189-96.

[33] Radomsky ML, Aufdemorte TB, Swain LD, Fox WC, Spiro RC, Poser JW. Novel formulation of fibroblast growth factor-2 in a hyaluronan gel accelerates fracture healing in nonhuman primates. J Orthop Res 1999;17:607-14.

[34] Kohn J, Abramson S, Langer R. Bioresorbable and bioerodible materials. In: Ratner BD, Hoffman AS, Schoen FJ, Lemons JE, editors. Biomaterial science: an introduction to materials in medicine. London: Elsevier Academic Press; 2004.

[35] Mabilleau G, Stancu IC, Honoré T, Legeay G, Cincu C, Baslé MF, et al. Effects of the length of crosslink chain on poly(2-hydroxyethyl methacrylate) (pHEMA) swelling and biomechanical properties. J Biomed Mater Res A 2006;77:35-42. 